\title{
Proposta de intervenção visando à implantação de protocolos clínicos nos serviços hospitalares
}

\author{
Intervention proposal aimed at the implementation of clinical protocols in hospital services \\ Propuesta de intervención dirigida a la implementación de protocolos clínicos en servicios \\ hospitalarios
}

Eufanio Estefano Saqueti ${ }^{1 *}$, Lena Vânia Carneiro Peres ${ }^{1}$.

\begin{abstract}
RESUMO
Objetivo: Propor uma intervenção planejada com a utilização de planejamento estratégico situacional (PES) para viabilizar a reimplantação do protocolo de cirurgia segura (PCS) e melhorar os resultados dos serviços hospitalares. Métodos: Trata-se de uma pesquisa aplicada com abordagem qualitativa realizada nos moldes de uma situação-problema em que os conhecimentos pré-adquiridos foram utilizados para viabilizar a reimplantação do PCS com base na identificação das principais causas modificáveis e descritas nos nós críticos, pelos atores envolvidos diretamente no processo de aplicação do PCS. Resultados: Com a utilização do PES, foi possível alinhar conhecimento técnico de maneira organizada, didática, acessível com as melhores evidências científicas, e viabilizar a reimplantação do PCS de modo que garanta a segurança do paciente cirúrgico. Conclusão: Produzir conhecimento científico foi fundamental para a obtenção do sucesso na proposta de reimplantação do PCS na instituição. Portanto, de forma inequívoca o PES pode ser uma ferramenta acessível, viável e eficaz para implantação de protocolos clínicos nos serviços hospitalares.
\end{abstract}

Palavras-chave: Planejamento estratégico, Diagnóstico situacional, Protocolo de segurança, Segurança do paciente.

\begin{abstract}
Objective: To propose a planned intervention with the use of strategic situational planning (PES) to enable the reimplantation of the safe surgery protocol (PCS) and improve the results of hospital services. Methods: This is an applied research with a qualitative approach carried out along the lines of a problem situation in which the pre-acquired knowledge was used to enable the re-implantation of the PCS based on the identification of the main modifiable causes and described in the critical nodes, by actors directly involved in the PCS application process. Results: With the use of PES, it was possible to align technical knowledge in an organized, didactic, accessible way with the best scientific evidence, and to enable the reimplantation of the PCS in order to guarantee the safety of the surgical patient. Conclusion: Producing scientific knowledge was essential for achieving success in the proposal to reimplant the PCS in the institution. Therefore, unequivocally, PES can be an accessible, viable and effective tool for the implementation of clinical protocols in hospital services.
\end{abstract}

Keywords: Strategic planning, Situational diagnosis, Safety protocol, Patient safety.

\section{RESUMEN}

Objetivo: Proponer una intervención planificada con el uso de la planificación estratégica situacional (PES) para permitir el reimplante del protocolo de cirugía segura (PCS) y mejorar los resultados de los servicios hospitalarios. Métodos: Se trata de una investigación aplicada con abordaje cualitativo que se lleva a cabo en la línea de una situación problema en la que se utilizó el conocimiento pre-adquirido para posibilitar la reimplantación del PCS a partir de la identificación de las principales causas modificables y descrito en los nodos críticos, por los actores directamente involucrados en el proceso de solicitud del PCS. Resultados: Con el uso de PES, fue posible alinear los conocimientos técnicos de forma organizada, didáctica, accesible con la mejor evidencia científica, y posibilitar el reimplante del PCS de forma que se garantice la seguridad del paciente quirúrgico. Conclusión: Producir conocimiento científico fue fundamental para lograr el éxito en la propuesta de reimplantar el PCS en la institución. Por tanto, de forma inequívoca, los PES pueden ser una herramienta accesible, viable y eficaz para la implementación de protocolos clínicos en los servicios hospitalarios

Palabras clave: Planificación estratégica, Diagnóstico situacional, Protocolo de seguridad, Seguridad del paciente.

1 Universidade Municipal de São Caetano do SUL (USCS), São Caetano do Sul - SP.

*E-mail: eufanio.saqueti@hotmail.com

SUBMETIDO EM: 4/2021

ACEITO EM: 5/2021

PUBLICADO EM: 6/2021 


\section{INTRODUÇÃO}

A elevada incidência de danos aos pacientes durante a assistência à saúde, tem sensibilizado a Organização Mundial de Saúde (OMS) e os sistemas de saúde em todo o mundo a melhorar a segurança do paciente (BRASIL, 2014; BRASIL, 2017).

A ocorrência de incidentes relacionados à assistência à saúde, e em particular os eventos adversos (EA) são questões relevantes, desde a publicação do relatório "Errar é Humano", pelo Institute of Medicine (IOM), em 1999, ao apontar que há significativa ocorrência de eventos adversos e que mais da metade dessas ocorrências eram ocasionadas por erro médico (TRAVASSOS C, 2012; BRASIL, 2017; FURINI ACA, et al., 2019).

Em países desenvolvidos estudos estimam que de 4,0\% a 16\% de pacientes hospitalizados são afetados por erro humano (BRASIL, 2017). Estima-se que o erro humano em procedimentos cirúrgicos ocorre em 10\% das intervenções cirúrgicas, chegando a 234 milhões de cirurgias ao ano (uma cirurgia para cada 25 pessoas vivas). Destas, $50 \%$ poderiam ser evitadas (BRASIL, 2014).

No biênio 2007-2008, o foco na cirurgia segura foi o desafio global escolhido. Foram definidas quatro ações importantes: I prevenção de infecções em sítios cirúrgicos; II anestesia segura; III equipes cirúrgicas seguras; e IV indicadores da assistência cirúrgica (BRASIL, 2013).

Sistemas mal desenhados produzem resultados ruins. Para modificar esta realidade nos ambientes organizacionais de assistência médica é necessário evitar o erro (BRASIL, 2014). De acordo com Reason J (2020) falhas humanas e técnicas, são impossíveis de serem eliminadas, porém os erros em saúde, podem ser evitados (SAQUETI EE, 2021).

No exercício da medicina, os erros médicos ocorrem, e suas consequências comprometem a segurança do paciente. Para melhorar a segurança do paciente é preciso mudar este cenário, identificando os erros, avaliando as causas e tomando medidas que reduzam a probabilidade de eventos adversos (MOTTA FILHO GR, et al., 2013).

Portanto, para que situações de risco sejam evitadas, se faz necessário oferecer assistência médicohospitalar com segurança e criar estratégias que antecipem, previnam e bloqueiem os erros antes de causarem danos (WATCHER RM, 2013).

A prática de protocolos clínicos (PC) como instrumentos para auxiliar na tomada de decisões e garantir um atendimento mais qualificado ao paciente tem chamado a atenção dos profissionais (médicos, especialistas ou clínicos) favoráveis à sua utilização, e estimulado o seu debate (SAQUETI EE, 2021).

Para alinhar a precisão técnica à segurança dos pacientes, em todos os níveis, o protocolo para cirurgia segura visa também, a redução dos efeitos adversos, a melhorar a qualidade da assistência prestada, conhecer os riscos e incidentes que ocorrem nas instituições brasileiras, direcionar o planejamento de ações dos gestores de saúde; melhorar a qualidade dos dados encaminhados e, por fim, reduzir custos com falhas e tempo extra de internações (CAPUCHO HC e CASSIANI SHB, 2013). Pois, falhas na segurança e riscos não controlados durante a assistência cirúrgica, muitas vezes, podem causar danos, irreparáveis aos pacientes (CORONA ARPD e OENICHE ACG, 2015).

Vincent C e Amalberti R (2016) defendendo a ideia de que, a segurança do paciente está relacionada com as tentativas de melhorar a confiabilidade de processos clínicos ou estratégias mais amplas de melhoria do sistema, argumentam que poucas são as estratégias destinadas a gerir os riscos nas condições cotidianas de trabalho no cuidado de saúde. Assim sendo, propõem que a segurança, implica o mapeamento dos riscos e dos benefícios do cuidado ao longo da trajetória do paciente pelo sistema de saúde.

É possível reduzir a ocorrência de erros e tornar os processos mais confiáveis aumentando a confiabilidade e desenvolvendo estratégias proativas para gerir continuamente os riscos em ambientes menos controlados (VINCENT C e AMALBERTI R, 2016). A implementação de iniciativas voltadas à segurança do paciente por meio de ações concretas para promoção do cuidado seguro são condições mínimas para o avanço na assistência segura (BRASIL, 2013; REIS GAX, et al., 2017; REIS GAX, et al., 2019). 
Porém, a institucionalização da segurança nas organizações de saúde, ainda é um processo cultural. A cultura de segurança no processo assistencial, ou seja, nas organizações de saúde, relatada na literatura evidencia que o desenvolvimento de boas práticas, isto é, de prática segura, encontra algumas barreiras e limitações no desenvolvimento de estratégias de segurança (REIS GAX, et al., 2019, OLIVEIRA RM, et al., 2014).

De acordo com Hull L (2017) as questões impactantes no sucesso dos protocolos de segurança concentram-se na sua implantação. Mitchell B, et al. (2017) relataram que apenas a padronização das práticas humanas não são suficientes para o êxito dos protocolos de cirurgia segura, sendo necessárias adaptações para o contexto local.

O Planejamento Estratégico Situacional (PES), desenvolvido por Carlos Matus, a partir dos anos 1970, apresenta-se como uma ferramenta de estratégia da saúde que atende às complexidades das situações existentes, focada nas constantes mudanças da situação real, para realizar análises situacionais, e mostrase ser um método adequado de planejamento governamental (BRASIL, 2014; LEMOS GS, et al., 2017).

$\mathrm{Na}$ saúde a capacidade de enfrentamento dos problemas existentes deve ser orientada por ações estratégicas bem definidas que visem a reduzir a incidência e prevalência de agravos à saúde do paciente cirúrgico. O PES, possui métodos e técnicas que se adequam aos mais diversos problemas da sociedade, e a partir da explicação situacional e do envolvimento de todos os atores, tem por finalidade fornecer estratégias de soluções (MORATO JAQ e SILVA BC, 2016).

De acordo com Rieg DL, et al. (2014) três características integram a composição do PES de Carlos Matus. A primeira é que na base do planejamento estão os problemas. A segunda apresenta o mundo de maneira subjetiva. A terceira reconhece que o futuro é incerto e incapaz de ser predito. Portanto, no PES de Carlos Matus um problema deve ser conhecido, compreendido e interpretado antes da construção de um plano.

Fundamentado nos momentos explicativo, normativo, estratégico e tático-operacional, o PES pode possibilitar a intervenção de maneira estratégica nos problemas ocorridos no ambiente das instituições de saúde (LEMOS GS, et al., 2017).

De modo geral, a literatura sobre PES sustenta que essa ferramenta pode ser um aliado na implementação das práticas de segurança nos pacientes cirúrgicos tendo em vista a necessidade de estratégia adequada, pautada em um diagnóstico situacional de cada hospital, envolvimento do maior número de pessoas possível que estão envolvidas no fluxo do paciente cirúrgico (GOMES CDPP, et al., 2016).

O PES se apresenta como um instrumento de planejamento útil na elaboração, formalização e implementação do Protocolo de Cirurgia Segura (PCS) uma vez que, serve para conduzir as ações do ator de forma efetiva em cada momento, para que sintomas e causas de um problema sejam erradicados. Portanto, torna-se um instrumento adequado para explicar realidades complexas (SAQUETI EE, 2021).

Neste contexto, fundamentado na cultura de segurança, este estudo objetivou propor uma intervenção planejada com a utilização de PES para viabilizar a reimplantação do PCS para melhorar os resultados dos serviços hospitalares.

\section{MÉTODOS}

A investigação foi delineada nos moldes da pesquisa aplicada com abordagem qualitativa objetivando propor uma intervenção planejada com a utilização de PES para viabilizar a reimplantação do PCS para melhorar os resultados dos serviços hospitalares.

O estudo foi realizado após aprovação do Comitê de Ética em Pesquisa da Universidade Municipal de São Caetano do Sul (USCS) conforme Parecer Consubstanciado número 4.162.897, e Certificado de Apresentação e Apreciação Ética (CAAE) número 34729220.4.0000.5510. O estudo respeitou as diretrizes e critérios estabelecidos na Resolução 466/12 do Conselho Nacional de Saúde (CNS), os preceitos éticos foram considerados em todo o processo de construção do trabalho. Como critérios de inclusão os participantes foram voluntários profissionais de saúde envolvidos em todo o processo de execução do PCS e que aceitaram participar do estudo e concordaram com o TCLE. 
Foram excluídos do estudo profissionais que se recusaram a participar do estudo e que se recusaram a concordar com o TCLE. Qualquer condição do participante que limite a capacidade para participar do estudo e, profissionais de saúde que não estejam envolvidos no processo de execução do PCS e se recusem a participar. Foi garantido o anonimato dos participantes, o sigilo das informações coletadas e garantido 0 desejo de interrupção do estudo a qualquer momento.

Fundamentado na cultura de segurança estabeleceu-se como propósito atender a necessidade de reimplantar o PCS em um hospital localizado no município de Campo Mourão-PR utilizando o PES. O início dos trabalhos foi conduzido seguindo os vértices do triângulo de Carlos Matus (Figura 1). A opção pelo PES para o desenvolvimento da intervenção planejada se justifica pelo fato de comportar categorias de análise que se adaptam ao conteúdo da proposta de construção de Protocolo de Cirurgia Segura.

Em organizações complexas como no caso da saúde, contar com um método de planejamento é imprescindível. Foi pensando sobre a necessidade de aumentar a capacidade de governar que Carlos Matus concebeu o PES, um método de planejamento centrado em problemas e em operações que deverão ser efetuadas para saná-los, com apoio de um ator social cuja ação e situação formam um todo complexo (LACERDA JT de, et al., 2013).

Figura 1 - Interfaces dos três extremos do triângulo de Carlos Matus.

\section{TRIÂNGULO DE GOVERNO}

\section{MATUS}

\section{PROJETO}

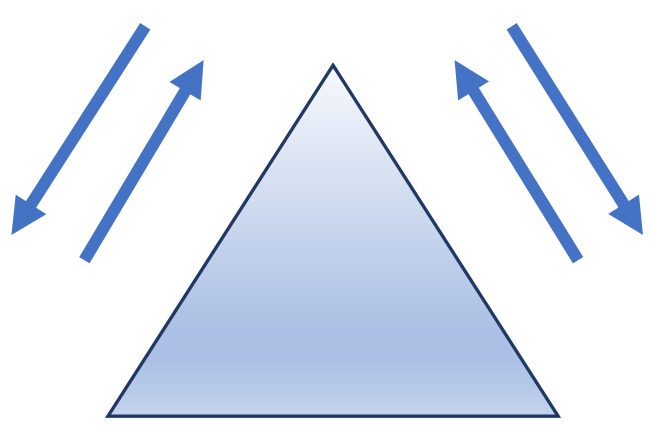

GOVERNABILIDADE

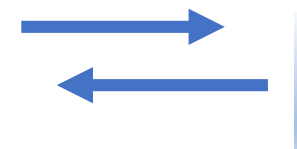

\section{CAPACIDADE DE GOVERNO}

Fonte: Saqueti EE e Peres LVC, 2021.

A coleta de dados ocorreu entre julho e agosto de 2020 no próprio hospital em horário de expediente com divisões dos profissionais dos setores do hospital, para que o atendimento ao usuário transcorresse sem prejuízos. A composição do grupo de trabalho foi com participantes voluntários profissionais envolvidos em todo o processo de aplicação do próprio PCS.

Na Oficina realizada presencialmente com autorização do Diretor Presidente do Hospital, o grupo de trabalho, em quatro encontros com duração de três horas cada, utilizando de tarjetas trabalhou de forma democrática para desenvolver os momentos explicativo, normativo e estratégico. Cada um dos momentos foi realizado com a participação de todos os atores envolvidos diretamente no processo de aplicação do PCS, para analisar os mais diversos problemas existentes e as possibilidades para solucioná-los. 


\section{Momento explicativo}

Momento da identificação dos problemas e seleção dos mais relevantes, realizado de modo a se obter um conjunto de fluxograma analítico (autoanálise), com o qual foi possível fazer o diagnóstico situacional, identificar os nós críticos, para possibilitar traçar estratégias e ações, calcular riscos e operacionalizar soluções.

Os participantes identificaram como problemas na aplicação do PCS os seguintes pontos: falta de treinamento dos médicos, organização da equipe medica, falta de adesão médica, internação em cima da hora, falta de comprometimento da equipe, acredito que não pensam na importância, só mais um papel a ser preenchido, profissionais acham que não tem importância, baixa adesão em treinamentos sobre o assunto, falta de conhecimento sobre a importância do protocolo, falta de treinamento periódico (funcionários), falta de conhecimento do protocolo, falta de treinamento multiprofissional, falta de conhecimento e responsabilidade no preenchimento, não tem indicador de preenchimento, não são aplicadas barreiras pelo não preenchimento, falta de recursos humanos, pressão para realizar o procedimento.

Cada apontamento foi discutido, agrupado e selecionado com base em seu aspecto mais relevante. Foram destacados os pontos principias, e identificados alguns macroproblemas. Em seguida, a interligação dos macroproblemas com base no princípio "do que leva ao que" de Carlos Matus, possibilitou identificar os nós críticos (Figura 2).

Figura 2 - Fluxograma nós críticos.
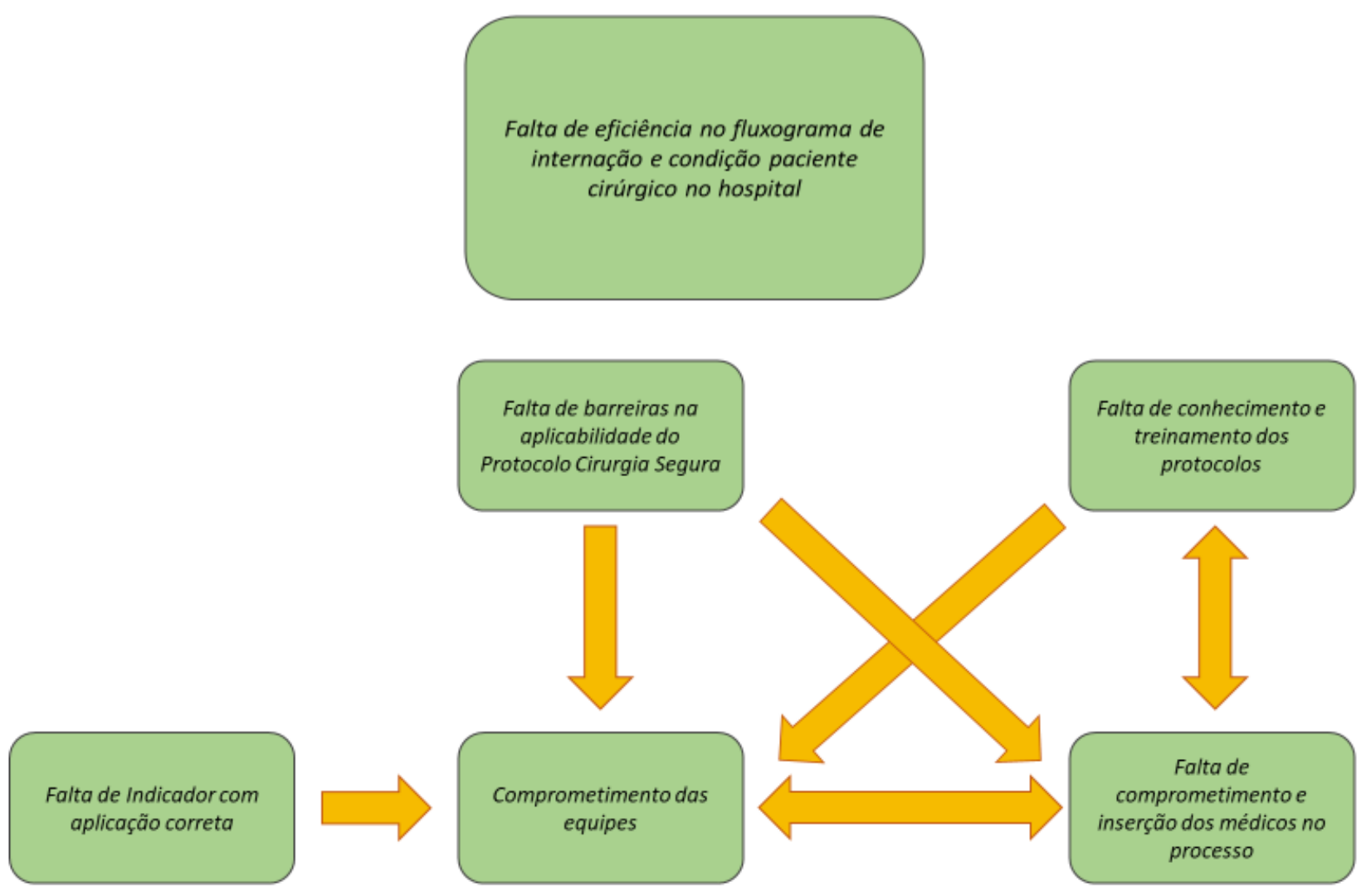

Fonte: Saqueti EE e Peres LVC, 2021.

\section{Momento normativo}

Após os nós críticos ficarem interligados, de forma consensual, iniciou-se o momento normativo. Nesta fase, cada integrante do grupo de trabalho sugeriu de forma aleatória algumas soluções que resolvessem de forma individual ou conjunta os nós críticos escolhidos (Figura 3). 
Figura 3 - Fluxograma momento normativo: sugestões para os nós críticos.

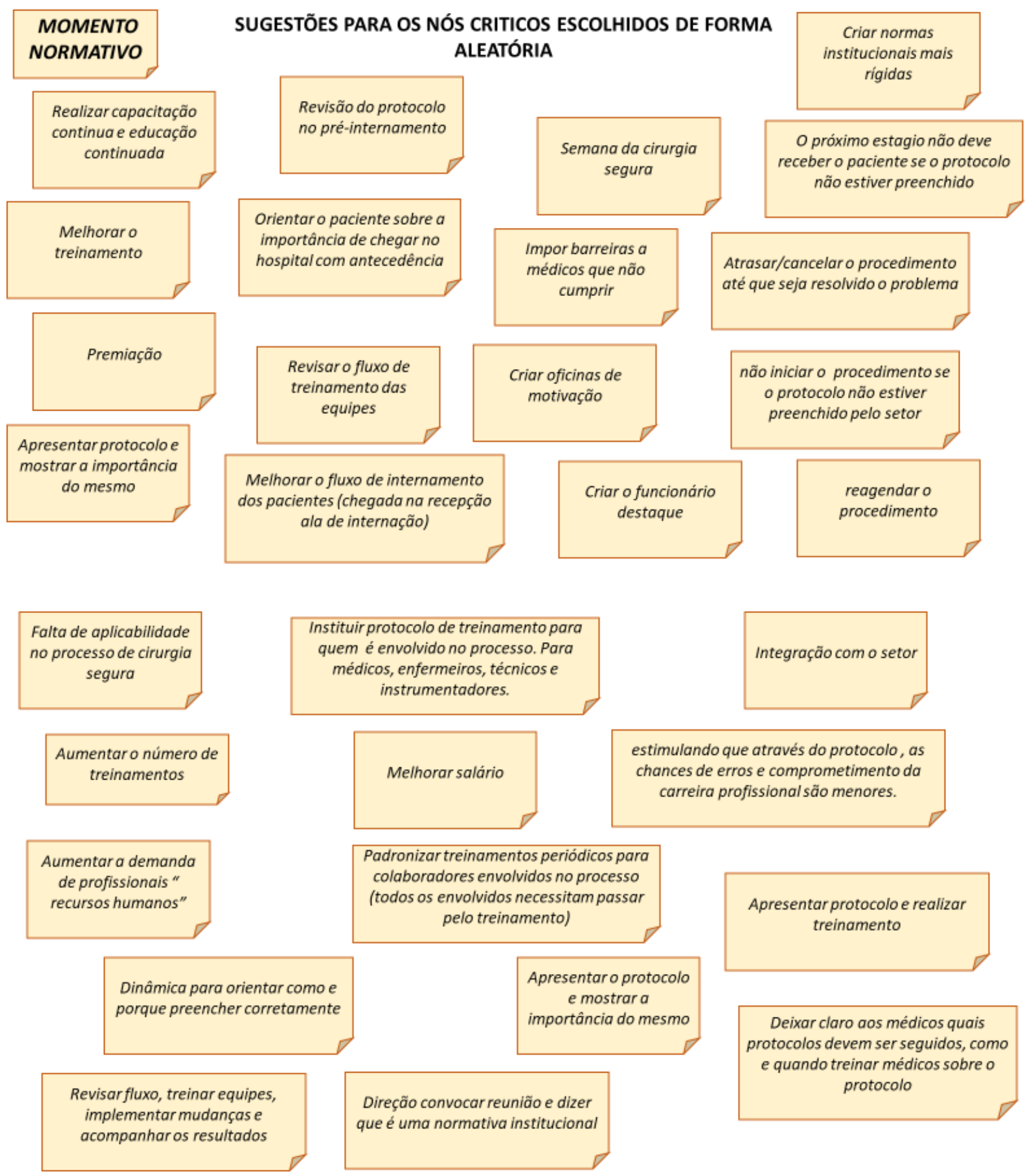

Fonte: Saqueti EE e Peres LVC, 2021.

Todas as sugestões de soluções propostas por cada integrante do grupo de trabalho foram listadas e agrupadas dentro de cada nó crítico selecionado no momento explicativo. Como ação final do momento normativo, foram reavaliadas todas as sugestões, ponderando de forma clara e democrática sobre a viabilidade de execução e a governabilidade para as mesmas, o que possibilitou chegar à versão final de soluções para cada nó crítico, encerrando esta fase do PES (Figura 4). 
Figura 4 - Fluxograma PES: validação final.

VALIDAÇÃO FINAL

FALTA DE COMPROMETIMENTO DAS EQUIPES

- melhorar a motivação dos colaboradores

- realizar semana cirurgia segura
FALTA DE COMPROMETIMENTO E INSERÇÃO DOS MEDICOS NO PROCESSO

treinar e apresentar aos médicos o Protocolo de cirurgia segura

- fazer cumprir o protocolo de cirurgia segura.

FALTA DE BARREIRAS NA APLICABILIDADE DO PCS

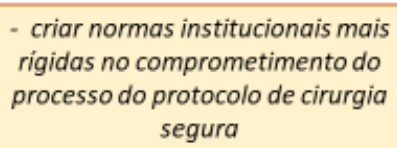

- criar normas institucionais mais rígidas no comprometimento do processo do protocolo de cirurgia segura

\section{FALTA DE CONHECIMENTO E TREINAMENTO DOS PROTOCOLOS}

educação continuada do protocolo de cirurgia segura integração das equipes com protocolo de cirurgia segura - integração dos novos colaboradores nos setores

\section{FALTA DE EFICIENCIA NO FLUXOGRAMA DE INTERNAÇAO E CONDUÇÃO DO PACIENTE CIRURGICO NO HOSPITAL}

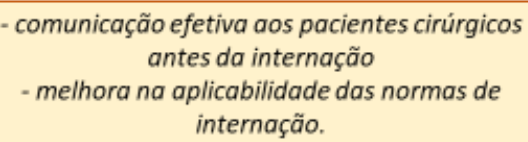

\section{Fonte: Saqueti EE e Peres LVC, 2021.}

Após a validação final do período normativo, passou-se a discutir as estratégias de execução das soluções possíveis apontadas no sentido de reimplementar o PCS na instituição. A ferramenta 5W2H foi a opção escolhida para auxiliar a aplicação eficiente destas estratégias. Para cada nó crítico e soluções apontadas pelo grupo, durante a oficina, foi trabalhado o que, porque, como, onde, quem, quando e quanto.

\section{RESULTADOS}

O diagnóstico situacional do PCS no Hospital em questão foi fundamental para o levantamento dos principais nós críticos. Os princípios do PES de Carlos Matus corroboram com essa necessidade (BRASIL, 2009).

Os nós críticos apontados pela equipe de trabalho foram: falta de barreiras na aplicabilidade do PES, falta de comprometimento das equipes, falta de conhecimento e treinamento dos protocolos, falta de comprometimento e inserção dos médicos no processo e falta de eficiência no fluxograma de internação e condução do paciente cirúrgico no hospital. Observa-se que são condições básicas e simples de serem apontadas quando da ineficiência da implantação de um protocolo clínico.

O grupo de trabalho considera que a superação dos nós críticos citados minimiza o insucesso, pois reimplantar o PCS com propriedade é uma das questões mais impactantes para a eficiência do PCS (HULL L, et al., 2017).

Todos esses nós críticos, receberam proposições de soluções que bastam à adesão voluntária da equipe para conferir êxito ao processo. No entanto, foi possível observar que quando algo não está em conformidade, há uma obrigatoriedade de atuação. Como exemplo, tem-se a criação de barreiras institucionais de interrupção do fluxo assistencial. Desta forma, o grupo atende ao observado por Criado JVN, et al. (2017) que consideram a adesão das equipes de forma voluntária e obrigatória como as maneiras mais efetivas.

Estratégias simples como a criação de barreiras de segurança e educação permanente já foram apontados por Oliveira RM, et al. (2014) como medida efetiva na implantação de PCS. Fato esse observado durante a oficina de trabalho sobre a falta de barreiras na aplicabilidade do protocolo em questão, propondo criar normas institucionais mais rígidas. Ou seja, uma vez que alguma fase no fluxo assistencial do paciente não tenha obedecido totalmente o PCS, o fluxo fica paralisado até que a pendência seja resolvida ou esclarecida. 
Conforme relatado por Mafra CR e Rodrigues MCS (2018) que atribuem à comunicação eficiente em ambiente cirúrgico como um excepcional benefício alcançado após o sucesso da implantação do PCS, a equipe PES aponta dois nós críticos a serem resolvidos, que podem melhorar a tão desejada comunicação entre os membros das equipes cirúrgicas, que são: inserção dos médicos no processo do PCS e falta de comprometimento das equipes.

Objetivando melhorar a adesão médica, o grupo de trabalho propôs treinar e apresentar o PCS aos médicos e fazer cumprir o PCS através da governabilidade oferecida ao Núcleo de Segurança do Paciente (NSP) do hospital. O envolvimento direto da equipe médica na implantação pode melhorar os resultados (GILLESPIE BM e MARSAHLL A, 2015).

Conforme Leape LLMD (2014) a agilidade e a liderança são necessárias para que as tarefas definidas sejam correta e eficientemente aplicadas na assistência a cada fase no atendimento de cada paciente cirúrgico. Novamente a equipe de trabalho tem um nó critico a ser superado para atingir esta expectativa (falta de eficiência no fluxograma de internação e condução do paciente cirúrgico no hospital).

O uso do PES na área da saúde já não é novidade, há registros em vários estudos e pesquisas identificando precisamente o ensinamento de Carlos Matus sendo utilizado para o enfrentamento de problemas nesse campo (JESUS WLA e ASSIS MMA, 2011). Porém, entende-se que sua utilização para implantação de protocolos clínicos e, especificamente PCS, é uma inovação no uso do PES respeitando os vértices do triângulo de Carlos Matus.

Utilizando o PES foi possível desenvolver um projeto de reimplantação do PCS na instituição. Interessante observar, que todos os atores envolvidos naturalmente participaram da construção dessa ferramenta dentro dos seus conhecimentos prévios sobre o fluxo do paciente cirúrgico, mas o projeto apresentado mostra a governabilidade das ações propostas, que surgiram de maneira natural nas discussões realizadas durante a Oficina.

Outra constatação fundamental, é que a implementação do plano de trabalho, chamado de momento tático operacional, ocorrerá com o envolvimento dos próprios atores, uma vez que eles são as personagens centrais em todo o fluxo do paciente cirúrgico na instituição.

Desta forma observa-se que: se o PES for aplicado desde o seu início, com a escolha ou eleição dos atores de forma que tenham envolvimento no fluxo assistencial do paciente operatório, um projeto construído com governabilidade e capacidade de governo acontecendo naturalmente, respeitando assim o triângulo de Carlos Matus, poderá ser implantado.

Vale ressaltar que o NSP, estava representado nas oficinas do PES e contava com a anuência e obrigação emitida pela diretoria do hospital, para implantar o PCS na instituição em questão. Portanto, o PCS tem na instituição um projeto de implantação, que será executado pelo NSP (Governabilidade) com os profissionais da área de saúde dos mais diversos setores que também contará com representantes nas oficinas do PES (Capacidade de governo).

Isso referenda que o PES é uma ferramenta a ser utilizada pelas instituições hospitalares na implementação de protocolos clínicos, mas com o cuidado extremo de constituir o grupo de trabalho com atores que estejam envolvidos diretamente na assistência dos pacientes que serão submetidos a estes protocolos. Pois esses atores além de construírem um projeto de implantação, saberão o que fazer em suas funções específicas contribuindo para o sucesso do mesmo e também irão construir um projeto alinhado com as necessidades, pensamento dos diretores e objetivos da instituição.

Enfim, pode-se destacar que a pesquisa gera conhecimento sobre situações de risco. Pois demonstra de forma clara que os profissionais inseridos no atendimento do hospital em questão ainda carecem de conhecimento sobre o PCS, instrumento fundamental para o sucesso na assistência dos pacientes cirúrgicos.

Porém, a identificação de uma ferramenta viável e eficiente na implantação de protocolos clínicos nas mais diversas instituições hospitalares, pode tornar-se uma rotina no trabalho árduo de planejamento estratégico. Pois o diagnóstico situacional como pré-requisito de aplicabilidade do mesmo torna a expectativa de sucesso 
algo visível e principalmente vivido por todos os atores envolvidos. Envolver pessoas ligadas diretas ao trabalho amiúde de seus setores no próprio planejamento, permite uma maior adesão no momento prático de aplicação no que se deseja. Obviamente que os melhores resultados significam menos morbimortalidades no atendimento médico hospitalar.

O fator limitador de aplicabilidade do PES na implantação de protocolos clínicos está pautado em projetos sem envolvimento das pessoas corretas, ausência de governabilidade e até de capacidade de governo das propostas, o que pode tornar a implantação dos protocolos inatingíveis.

\section{CONCLUSÃO}

Dado o exposto, pode-se afirmar que os resultados confirmam que para o PCS ser um mecanismo de transformação da prática cirúrgica é preciso que uma política de segurança e um sistema de monitoração e gestão dos processos organizacionais sejam conduzidos efetivamente, para que o ato cirúrgico possa refletir na melhoria de indicadores, após sua reimplantação. A segurança do paciente durante a assistência cirúrgica, dever ser implementada e monitorada com o mesmo rigor em todos os serviços de saúde, tal como uma política de governo, de acordo com o que foi evidenciado na literatura. Portanto, conclui-se de forma inequívoca que o PES pode ser uma ferramenta acessível, viável e eficaz para implantação de protocolos clínicos nos serviços hospitalares.

\section{REFERÊNCIAS}

1. BRASIL. Agência Nacional de Vigilância Sanitária - ANVISA. Segurança do paciente. Brasília - DF. 2017.

2. BRASIL. Agência Nacional de Vigilância Sanitária - ANVISA. Assistência segura: uma reflexão teórica aplicada à prática. Brasília - DF. 2013.

3. BRASIL. Ministério da Saúde. Documento de referência para o Programa Nacional de Segurança do Paciente. Ministério da Saúde; Fundação Oswaldo Cruz; Agência Nacional de Vigilância Sanitária. Brasília: Ministério da Saúde, 2014.

4. CAPUCHO HC, CASSIANI SHB. Necessidade de implantar programa nacional de segurança do paciente no Brasil. Rev Saúde Pública 2013; 47(4): 791-8

5. CORONA ARPD, PENICHE ACG. A cultura de segurança do paciente na adesão ao protocolo da cirurgia segura. Rev. Sobecc, São Paulo. Jul./Set. 2015; 20(3): 179-185

6. CRIADO JVN, et al. Processo de implementação do protocolo de cirurgia segura. Revista Cubana de Enfermería, 2017; 33(1).

7. FURINI ACA, et al. Notificação de eventos adversos: caracterização dos eventos ocorridos em um complexo hospitalar. RGE Revista Gaúcha de Enfermagem. 2019;40 (esp).

8. GILLESPIE BM, MARSHALL A. Implementação de listas de verificação de segurança em cirurgia: uma síntese realista de evidências. Implementation Sci, 2015; 10(137).

9. GOMES CDPP, et al. Percepção de uma equipe de enfermagem sobre a utilização do checklist cirúrgico. Rev. Sobecc, São Paulo, jul./set. 2016; 21(3): 140-145.

10. HULL L, et al. Ciência de Implementação: uma oportunidade negligenciada para acelerar melhorias na segurança e qualidade do atendimento cirúrgico Annals of Surgery. Jun. 2017; 265(6): 1104-1112.

11. JESUS WLA, ASSIS MMA. Desafios do planejamento na construção do SUS. Salvador: EDUFBA, 2011. $174 \mathrm{p}$

12. LEAPE LLMD. O enigma da lista de verificação. The New England Journal of Medicine, 2014; 370: 1063-1064.

13. LEMOS GS, et al. Adoção do planejamento estratégico situacional na farmacovigilância e segurança do paciente de um projeto ensino-serviço. Rev. Eletr. Farm., Goiânia, 2017; 14(4): 5-16.

14. MAFRA CR, RODRIGUES MCS. Lista de verificação de segurança cirúrgica: Uma revisão integrativa sobre benᄀefícios e sua importância. Rev. Fund Care Online. jan./mar. 2018; 10(1): 268-275.

15. MITCHELL B, et al. Está se mobilizando ou parado? Uma revisão narrativa do conhecimento da Lista de Verificação de Segurança Cirúrgica desenvolvida em 25 artigos altamente citados de 2009 a 2016. BMJ Quality \& Safety, 2017; 26: 837844

16. MORATO JAQ, SILVA BC. Planejamento estratégico situacional: um instrumento para formação e Gestão de políticas públicas. Revista Gestão Pública: práticas e desafio RGPD, OUT. 2016; x(2).

17. MOTTA FILHO GR, et al. Protocolo de cirurgia segura da OMS: o grau de conhecimento dos ortopedistas brasileiros. Rev. Bras. Ortop. 2013; 48(6): 554-562.

18. OLIVEIRA RM, et al. Estratégias para promover segurança do paciente: da identificação dos riscos às práticas baseadas em evidências. Esc. Anna Nery. Rio de Janeiro, Jan./Mar. 2014; 18(1).

19. REIS GAX, et al. Dificuldades para implantar estratégias de segurança do paciente: perspectivas de enfermeiros gestores. Rev Gaúcha Enferm. 2019; 40(esp): e20180366.

20. REIS GAX, et al. Nurse manager perceptions of patient safety strategy implementation. Texto Contexto Enferm. 2017; 26(2): e00340016.

21. RIEG DL, et al. Aplicação de procedimentos do planejamento estratégico situacional (PES) para estruturação de problemas no âmbito empresarial: estudos de casos múltiplos. Gest. Prod., São Carlos, 2014; 21(2): 417-431.

22. SAQUETI EE. Utilização do planejamento estratégico situacional para a institucionalização de protocolo de cirurgia segura na Santa Casa de Campo Mourão-PR. Dissertação (Mestrado em Saúde) - São Caetano do Sul - USCS, $2021 ; 126$ p.

23. TRAVASSOS C. Segurança do paciente doente, o que é? Curso Introdutório - Investigação em Segurança do Paciente. World Health Organization, 2012.

24. VINCENT C, AMALBERTI R. Cuidado de saúde mais seguro: estratégias para o cotidiano do cuidado. Rio de Janeiro, 2016.

25. WATCHER RM. Compreendendo a segurança do paciente. 2. ed. Porto Alegre: Artmed; 2013. 